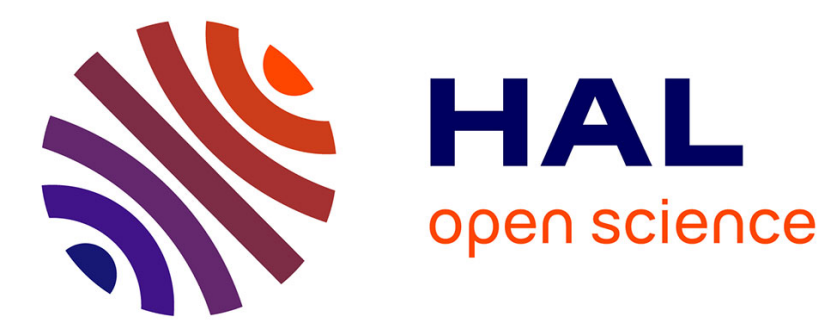

\title{
Effects of an electromagnetic quark form factor on meson properties
}

B. Silvestre-Brac

\section{To cite this version:}

B. Silvestre-Brac. Effects of an electromagnetic quark form factor on meson properties. Nuclear Physics A, 2002, 712, pp.303-326. 10.1016/S0375-9474(02)01288-5 . in2p3-00011931

HAL Id: in2p3-00011931

https://hal.in2p3.fr/in2p3-00011931

Submitted on 25 Nov 2002

HAL is a multi-disciplinary open access archive for the deposit and dissemination of scientific research documents, whether they are published or not. The documents may come from teaching and research institutions in France or abroad, or from public or private research centers.
L'archive ouverte pluridisciplinaire HAL, est destinée au dépôt et à la diffusion de documents scientifiques de niveau recherche, publiés ou non, émanant des établissements d'enseignement et de recherche français ou étrangers, des laboratoires publics ou privés. 


\title{
Effects of an electromagnetic quark form factor on meson properties
}

\author{
B. Silvestre-Brac ${ }^{\text {a }}$ \\ ${ }^{a}$ Institut des Sciences Nucléaires, IN2P3, CNRS, Université Joseph Fourier, 53 \\ Av. des Martyrs, 38026 Grenoble Cedex, France
}

\begin{abstract}
A form factor is introduced in the quark electromagnetic current. Its effect is analyzed on charge mean square radii and form factors in the mesonic sector. The decay of a vector meson into lepton-antilepton pair is also affected. Two different expressions for the form factors, and two different types of quark potential are tested and some relativistic kinematical corrections are proposed. In any case the introduction of a quark form factor greatly improves the agreement with experimental data.
\end{abstract}

\section{Introduction}

Quantum Chromodynamics (QCD) is believed to be the good theory for strong interactions. However, its application to the so-called non perturbative regime is far from being obvious. Even in the mesonic sector where only a valence quark-antiquark pair is effective, a basic theory, such as lattice QCD, is very cumbersome. Very often, people prefer to use some realistic models (named QCD inspired) to describe the same physics. Among them, the non-relativistic quark model (NRQM) is very attractive [1]-[2]. It is simple enough in the mesonic sector since the solution of the resulting Schrödinger equation is easily obtained numerically; moreover the center of mass motion can be treated exactly. Also, the wave function has an immediate quantum mechanical interpretation. Lastly, it has met with many successes, even in domains where it should fail in principle. A slight modification is sometimes invoked : the introduction of a relativistic expression for the kinetic energy operator[3]. The resulting equation is called a Salpeter equation. Although it is more complicate to solve than an ordinary Schrödinger equation, the developed numerical algorithms are nowadays very efficient and handling a Salpeter equation is not really a problem [4].

In NRQM, the effective quark degrees of freedom are the constituent quarks; 
they have the same quantum numbers as the current quarks of the basic theory, but differ by other properties. They are dressed by gluon and quark-antiquark pairs; in particular the constituent mass is significantly different from the current mass. From the dynamical point of view, quark-antiquark potentials used in Schrödinger or Salpeter equations have reached a high degree of sophistication. They include the dressing of the quarks and the resulting potential is the convolution of the bare potential with the gluonic density of the quark. The spectra of mesons, except some typical states that need other interpretation, are nicely reproduced [5]-[6]. Chiral symmetry is a basic symmetry of QCD lagrangian for quarks with zero mass. This symmetry is broken as soon as constituent masses are used, as in a Schrödinger or a Salpeter equation.

Always with the same underlying philosophy that quarks inside hadrons, as used in NRQM models (and many others in fact), must be considered as quasiparticles, there is no reason to maintain their electromagnetic properties identical to those of the bare quarks. However, since they originate from the electromagnetic and not the strong interactions, the corresponding densities should be different. The theory is not so advanced as to obtain the gluonic or electromagnetic density directly from first QCD principles. Thus, both must be postulated on phenomenological grounds. Curiously, modifications due to electromagnetic quark density are very rarely taken into account. I think that one reason for that is historical. At the beginning of the quark model, one famous success was the explanation of proton and neutron magnetic moments in a very simple way, without need for sophisticated corrections. But other electromagnetic observables cannot be described with such simple models. Chiral symmetry violations presumably originate from strong interactions although they can have indirect manifestations on electromagnetic properties through the wave function. Here this problem will not be addressed, accepting basically that chiral symmetry is broken from the very beginning in the NRQM.

The aim of this paper is the study of some electromagnetic meson properties resulting from the introduction of an electromagnetic quark form factor. Precisely, I will focus my analysis on the meson form factors and on the decay of vector mesons into lepton-antilepton pairs. I have in mind to see whether a consistent scheme is able to explain both observables.

In the next section, I describe the potentials used for obtaining the meson wave functions. In the third section the electromagnetic quark current and its modification are discussed. In the fourth and the fifth sections I focus on the description of meson form factors and leptonic decays. The sixth section is devoted to the results and their comments and the conclusions are drawn in the last section. In the appendix, I give elements to calculate some physical quantities. 


\section{Meson wave function}

\subsection{Potentials}

I consider the meson as a two-body system, whose wave function $|\Psi\rangle$ is given by solving the usual equation :

$$
H|\Psi\rangle=E|\Psi\rangle
$$

where the hamiltonian $H$ is composed of two terms : a kinetic energy operator $T$ and a potential term $V$ :

$$
H=T+V
$$

The kinetic energy has either a traditional non-relativistic (NR) expression $T=\mathbf{p}^{2} / 2 \mu$ ( $\mu$ is the reduced mass of the system), in which case the equation (1) is named Schrödinger equation, or a relativistic (R) expression $T=$ $\sqrt{\mathbf{p}^{2}+m_{1}^{2}}+\sqrt{\mathbf{p}^{2}+m_{2}^{2}}-m_{1}-m_{2}$, in which case it is called a Salpeter equation. The potentials that I use for the calculation do not contain spin-orbit or tensor forces, so that spin and orbital angular momenta are good quantum numbers [5]. Nevertheless they result from the convolution of bare potentials with gaussian gluonic density. Precisely they are composed of 3 terms :

$$
V=V_{c}+V_{h y p}+V_{\text {inst }}
$$

In this equation, $V_{c}$ is a central part, $V_{\text {hyp }}$ an hyperfine term and $V_{\text {inst }}$ a term taking care of instantons. The central part contains asymptotic freedom fitted on the experimental value of $\alpha_{s}\left(Q^{2}\right)$ and a linear confining term. The hyperfine term is a sum of two gaussian forms, one with long range and the other with short range. Its effects is to split the levels with different spin values. The term $V_{\text {inst }}$ is short range and is effective only for spin zero states. The main property of this term is to mix different flavour channels and in particular it allows to explain the $\eta$ sector. The various parameters entering the potential depend of course on which type of kinetic energy operator is retained, and have been fitted on the meson spectra. In this paper I will consider two different potentials : DNR which is based on a non-relativistic kinetic energy operator, and DSR which is based on a relativistic kinetic energy operator. Both give very nice spectra, but their wave functions can differ significantly and this is a good check for my purpose. The exact form, as well as the parameters, of these potentials have been given elsewhere [5]. 


\subsection{Meson wave function}

The numerical algorithm to solve the Salpeter equation was studied in several works [3][4]. Let me say that, in this paper and for both potentials, I expand the wave function as a sum of gaussian terms. I showed in many places that an expansion up to 5 terms is more than enough to get a very high accuracy on the wave function [7]-[8]. This is the prescription used for all calculated quantities in this paper. The advantage of such a procedure is that many quantities can be calculated analytically; moreover the Fourier transform of the wave function is very easy to obtain. This last advantage is of prime importance since, as you will see, in the present formalism, it is the momentum representation of the wave function that is more convenient. The practical calculation of important quantities is relegated to the appendix.

Since the formalism relies on quantum field theory, it is better to put the wave function in a second quantized form. Let me introduce the constituent quark (antiquark) creation operators $b_{\alpha}^{\dagger}(\mathbf{p}, s)\left(d_{\alpha}^{\dagger}(\mathbf{p}, s)\right)$; here $\alpha$ denotes the flavour as well as all intrinsic quantum numbers, except the spin projection explicitly denoted $s$, and $\mathbf{p}$ the momentum of the particle. In all what follows concerning the field quantities (spinors, normalization,...), I use the conventions of Bjorken-Drell [9]. In that scheme, the meson wave function is written :

$$
\begin{aligned}
|\alpha \beta, L S J M ; \mathbf{K}\rangle= & \sum_{\alpha_{1}, \beta_{2}}\left\langle\alpha \alpha_{1} \beta \beta_{2}|| J M\right\rangle(-1)^{1 / 2-s_{2}} \\
& \int d \mathbf{p}_{1} d \mathbf{p}_{2} \delta(\mathbf{P}-\mathbf{K}) \Psi(\mathbf{p}) b_{\alpha_{1}}^{\dagger}\left(\mathbf{p}_{1}, s_{1}\right) d_{\beta_{2}}^{\dagger}\left(\mathbf{p}_{2}, s_{2}\right)|0\rangle
\end{aligned}
$$

In this formula, the meson with quantum numbers $L S J M$ is composed of a quark of type $\alpha$ and antiquark of type $\beta$, coupled to good quantum numbers (colour singlet, spin, space angular momenta and isospin if necessary) with help of all necessary Clebsch-Gordan coefficients denoted symbolically by $\left\langle\alpha \alpha_{1} \beta \beta_{2}|| J M\right\rangle\left(\alpha_{1}\right.$ and $\beta_{2}$ mean all the magnetic quantum numbers for particles 1 and 2) and has a total linear momentum $\mathbf{K}$. As usual $\mathbf{P}=\mathbf{p}_{1}+\mathbf{p}_{2}$ is the total momentum and $\mathbf{p}=\left(m_{2} \mathbf{p}_{1}-m_{1} \mathbf{p}_{2}\right) /\left(m_{1}+m_{2}\right)$ the relative momentum of the system. To deal correctly with the rotational properties of the antiquark operator an extra phase $(-1)^{1 / 2-s_{2}}$ is needed. The meson as a whole is supposed to be in a plane wave state (denoted by the Dirac factor) and the intrinsic wave function $\Psi(\mathbf{p})$ (in principle it depends on all good quantum numbers but I forget this to have a simpler typographical notation) is expressed in momentum representation as :

$$
\Psi(\mathbf{p})=\varphi(p) Y_{L}^{\mu}(\widehat{p})
$$


with the normalization property:

$$
\int d \mathbf{p} \Psi^{*}(\mathbf{p}) \Psi(\mathbf{p})=1=\int_{0}^{\infty} d p p^{2} \varphi^{2}(p)
$$

Very often, when no ambiguity arises, I will simply note the wave function (4) as $\left|\Psi_{\mathbf{K}}\right\rangle$.

\section{Quark current operator}

The quark field for a flavour of type $\alpha$ is written traditionally in momentum representation as [9] :

$$
\begin{aligned}
q_{\alpha}(x)= & \sum_{s} \int \frac{d \mathbf{p}}{(2 \pi)^{3 / 2}} \sqrt{\frac{m_{\alpha}}{E_{p}(\alpha)}} \\
& \times\left[b_{\alpha}(\mathbf{p}, s) u_{\alpha}(\mathbf{p}, s) e^{-i p . x}+d_{\alpha}^{\dagger}(\mathbf{p}, s) v_{\alpha}(\mathbf{p}, s) e^{i p . x}\right]
\end{aligned}
$$

with usual definitions of the spinors $u$ and $v$ and $E_{p}(\alpha)=\sqrt{\mathbf{p}^{2}+m_{\alpha}^{2}}$. In non-relativistic approximations this last term is simplified into $m_{\alpha}$, but in the following I shall keep it as far as possible and I call the resulting modifications as kinematical relativistic corrections. The presence of this term implies that it is necessary to deal with a meson wave function in momentum representation.

The Dirac quark current is well known :

$$
j^{\mu}(x)=e \sum_{\alpha} e_{\alpha} \overline{q_{\alpha}}(x) \gamma^{\mu} q_{\alpha}(x)
$$

where $e$ is the proton charge and $e_{\alpha}$ is the fractional dimensionless quark charge. This expression is valid for point-like spin $1 / 2$ particles, like bare quarks. However I am interested in constituent quarks and to take care phenomenologically of the internal structure of these quasiparticles, I introduce electromagnetic form factors that modify the current in the following way :

$$
j^{\mu}(x)=e \sum_{\alpha} e_{\alpha} \overline{q_{\alpha}}(x) \Gamma_{\alpha}^{\mu} q_{\alpha}(x)
$$

The form of the modified operator $\Gamma_{\alpha}^{\mu}$ is written as usual [10]-[11] : 


$$
\begin{array}{r}
\overline{w_{\alpha}^{\prime}}\left(\mathbf{p}^{\prime}, s^{\prime}\right) \Gamma_{\alpha}^{\mu} w(\mathbf{p}, s)= \\
\overline{w_{\alpha}^{\prime}}\left(\mathbf{p}^{\prime}, s^{\prime}\right)\left[F_{1}^{\alpha}\left(q^{2}\right) \gamma^{\mu}+\frac{\kappa_{\alpha}}{2 m_{\alpha}} F_{2}^{\alpha}\left(q^{2}\right) i \sigma^{\mu v} q_{\nu}\right] w(\mathbf{p}, s)
\end{array}
$$

Depending upon the process, $w^{\prime}$ or $w$ means here either $u$ or $v$ and $q^{2}=q_{0}^{2}-\mathbf{Q}^{2}$ is the square momentum of the photon (zero for a real photon, but different from zero for a virtual photon). My convention is such that the exponential occurring in (9) is of the form $e^{i q . x}$. There exist a priori two independent form factors $F_{1}^{\alpha}\left(q^{2}\right)$ and $F_{2}^{\alpha}\left(q^{2}\right)$ with normalization conditions : $F_{1}^{\alpha}(0)=1=F_{2}^{\alpha}(0)$. In order to avoid too many free parameters I impose $F_{1}^{\alpha}\left(q^{2}\right)=F_{2}^{\alpha}\left(q^{2}\right)$ in all my applications; $\kappa_{\alpha}$ is the anomalous magnetic moment for the quark of flavour $\alpha$.

The expression (9) for the electromagnetic quark current is enough to obtain the meson form factor. For the decay of a vector meson into a lepton-antilepton pair, one needs the electromagnetic coupling operator that is defined as usual

$$
H_{e m}=\int d \mathbf{x}: j^{\mu}(x) A_{\mu}(x):
$$

where $A_{\mu}(x)$ is the photon field and : : denotes normal operator ordering. Now, since one deals with leptons, in addition to the quark current (9) one must consider the electromagnetic lepton current, so that the total current occurring in (11) should be :

$$
j^{\mu}(x)=e \sum_{\alpha} e_{\alpha} \overline{q_{\alpha}}(x) \Gamma_{\alpha}^{\mu} q_{\alpha}(x)-e \sum_{\beta} \overline{l_{\beta}}(x) \gamma^{\mu} l_{\beta}(x)
$$

\section{Meson form factor}

\subsection{Definition}

Experimentally, only pion and kaon form factors are known. So I consider form factors for pseudoscalar mesons $(L=0=S=J)$ only; thus the meson wave function (5) reduces in this case to $\Psi(\mathbf{p})=\frac{1}{\sqrt{4 \pi}} \varphi(p)$. The usual definition of the meson form factor $F_{M}\left(q^{2}\right)$ is (in this case only one form factor exists):

$$
\left\langle\Psi_{K^{\prime}}\left|j^{\mu}(0)\right| \Psi_{K}\right\rangle=e N\left(K^{\prime}+K\right)^{\mu} F_{M}\left(q^{2}\right)
$$

where $q=K^{\prime}-K$ and $N$ is a factor, which has different forms depending upon the authors [12]-[13]-[14], but which is related in fact to conventions in 
use (essentially normalization of plane waves, normalization of spinors and commutation properties of creation operators). By definition the meson form factor $F_{M}\left(q^{2}\right)$ is dimensionless and should verify $F_{M}(0)=e_{M}$ (the charge of the meson). Due to the fact that the wave function (4) is basically non relativistic and time independent, one cannot adopt a fully covariant definition as in (13). Moreover the experimental form factors are measured in the space-like region, and one defines $\mathbf{Q}^{2}=-q^{2}$ so that the definition of meson form factors in my formalism is :

$$
\left\langle\Psi_{\mathbf{K}^{\prime}}|\mathbf{j}(0)| \Psi_{\mathbf{K}}\right\rangle=e N\left(\mathbf{K}^{\prime}+\mathbf{K}\right) F_{M}\left(\mathbf{Q}^{2}\right)
$$

with $\mathbf{Q}=\mathbf{K}^{\prime}-\mathbf{K}$. I determine the normalization factor $N$ in such a way that, with the wave function $\left|\Psi_{\mathbf{K}}\right\rangle$ defined by (4) and the current $\mathbf{j}(0)$ defined by $(9-10), F_{M}(0)$ is the meson charge. More precisely, $N$ is determined by :

$$
\left\langle\Psi_{\mathbf{K}}|\mathbf{j}(0)| \Psi_{\mathbf{K}}\right\rangle=2 e\left(e_{\alpha}+e_{\beta}\right) N \mathbf{K}
$$

When calculating the matrix element of the current operator, there exist a contribution due to quark and another one due to antiquark; they are denoted respectively $\mathbf{j}_{q}$ (spinors $\bar{u} \Gamma u$ ) and $\mathbf{j}_{\bar{q}}$ (spinors $\bar{v} \Gamma v$ ).

Very often, people considered non-relativistic expression for the meson form factor. The theoretical curve deviates generally from the corresponding data. To cure this, some modifications, such that introduction of a kinematical boost , have been advocated. A large discussion on form factors and the proposed boost can be found in [15]. In this paper, I suggest that an equally good (and probably better) fit can be obtained by another mechanism, which seems to me more sound physically, namely an electromagnetic quark form factor.

\subsection{Non-relativistic approximation}

The non-relativistic (NR) approximation consists essentially by the replacement $E_{p}(\alpha) \longrightarrow m_{\alpha}$ both in the definition of fermion fields and in the Dirac spinors. The calculation of the $N$ factor by application of (15) is just a matter of straightforward calculation; one finds : $N=1 /\left[2(2 \pi)^{3}\left(m_{\alpha}+m_{\beta}\right)\right]$. The calculation of the form factor is more involved but presents no real difficulty; I quote here only the principal steps without entering into technical details.

With help of the Gordon identity, one can write the spinor matrix element appearing in $\mathbf{j}_{q}$ as :

$$
\overline{u_{\alpha}}\left(\mathbf{p}^{\prime}, s^{\prime}\right) \boldsymbol{\Gamma}_{\alpha} u_{\alpha}(\mathbf{p}, s)=
$$




$$
\delta_{s^{\prime} s} F_{1}^{\alpha}\left(q^{2}\right) \frac{\mathbf{p}^{\prime}+\mathbf{p}}{2 m_{\alpha}}+\left(F_{1}^{\alpha}\left(q^{2}\right)+\kappa_{\alpha} F_{2}^{\alpha}\left(q^{2}\right)\right) i(\sigma)_{s^{\prime} s} \times \frac{\mathbf{p}^{\prime}-\mathbf{p}}{2 m_{\alpha}}
$$

and a similar expression for the antiquark term in the current. Due to the scalar property of the wave function in spin space, the second factor, proportional to $(\sigma)_{s^{\prime} s}$, gives a vanishing contribution. Inserting the first factor in the expression of the wave function, dealing with the Dirac functions, one is left with an integral on a single variable, the relative momentum in the meson. This integral is composed of two parts : a term proportional to $\left(\mathbf{K}^{\prime}+\mathbf{K}\right)$ and a term which vanishes identically, due to the fact that the wave function $\varphi(p)$ depends only on the magnitude of $\mathbf{p}$ and not on its direction. Thus one is left with the term proportional to $\left(\mathbf{K}^{\prime}+\mathbf{K}\right)$, which must be identified to (14), giving the meson form factor. Explicitly ( $e_{\alpha}$ is the quark charge and $e_{\beta}$ is the antiquark charge),

$$
F_{M}\left(\mathbf{Q}^{2}\right)=e_{\alpha} F_{1}^{\alpha}\left(-\mathbf{Q}^{2}\right) I^{\alpha}\left(\mathbf{Q}^{2}\right)+e_{\beta} F_{1}^{\beta}\left(-\mathbf{Q}^{2}\right) I^{\beta}\left(\mathbf{Q}^{2}\right)
$$

where

$$
I^{\rho}\left(\mathbf{Q}^{2}\right)=\int d \mathbf{p} \Psi^{*}\left(\mathbf{p}+c_{\rho} \mathbf{Q}\right) \Psi(\mathbf{p}) ; \rho=\alpha, \beta
$$

and

$$
c_{\alpha}=\frac{m_{\beta}}{m_{\alpha}+m_{\beta}} ; c_{\beta}=-\frac{m_{\alpha}}{m_{\alpha}+m_{\beta}}
$$

Let us remark that the non-relativistic expression of the meson form factor depends only on the $F_{1}$ quark form factor. To get the traditional meson form factor obtained with point-like quarks (called bare form factor), it is sufficient to put $F_{1}^{\alpha}\left(-\mathbf{Q}^{2}\right)=1$ in $(17)$.

From the form factor, one gets the charge mean square radius of the meson by the definition $\left\langle r^{2}\right\rangle=-\left.6 \frac{d F_{M}\left(Q^{2}\right)}{d Q^{2}}\right|_{Q^{2}=0}$. Let us denote $H^{\rho}=-\left.6 \frac{d I^{\rho}\left(Q^{2}\right)}{d Q^{2}}\right|_{Q^{2}=0}$ and $G^{\rho}=-\left.6 \frac{d F_{1}^{\rho}\left(Q^{2}\right)}{d Q^{2}}\right|_{Q^{2}=0}$, the charge square radius can be written :

$$
\left\langle r_{N R}^{2}\right\rangle_{M}=\left\langle r_{N R}^{2}\right\rangle_{M}^{\text {bare }}+e_{\alpha} G^{\alpha}+e_{\beta} G^{\beta}
$$

where the bare charge square radius is simply given by :

$$
\left\langle r_{N R}^{2}\right\rangle_{M}^{\text {bare }}=e_{\alpha} H^{\alpha}+e_{\beta} H^{\beta}
$$

The quark form factor will be chosen as a decreasing function, so the $G^{\rho}$ 
quantities are always positive, and the effect of a quark form factor is to enhance the meson square radius.

\subsection{Relativistic approximation}

In this section, I want to keep the $E_{p}$ terms everywhere and avoid approximation, as far as I can. In fact this is not possible because the formalism is not always consistent and gets very complicated quite soon. This is the price to pay for using a non-relativistic wave function (4) in a quantum field expression for the current and the quark field. In the calculation of the normalization factor $N$, I must suppose that $K<<m_{\alpha}$ (but it is not necessary to have in addition $p<<m_{\alpha}$ as in the NR approximation). It is suitable to define a "relativized quark mass" $\widetilde{m}_{\alpha}$ (which coincides with the constituent mass $m_{\alpha}$ in the NR approximation) by the equation :

$$
\frac{1}{\widetilde{m}_{\alpha}}=\int d \mathbf{p} \frac{\Psi^{*}(\mathbf{p}) \Psi(\mathbf{p})}{E_{p}(\alpha)}
$$

and a "relativized charge" $\widetilde{e}_{\alpha}$ by :

$$
\widetilde{e}_{\alpha}=e_{\alpha} \frac{m_{\alpha}}{\widetilde{m}_{\alpha}}
$$

One can show that the normalization factor in this case is equal to :

$$
N=\frac{\widetilde{e}_{\alpha}+\widetilde{e}_{\beta}}{2(2 \pi)^{3}\left(m_{\alpha}+m_{\beta}\right)\left(e_{\alpha}+e_{\beta}\right)}
$$

The calculation of the meson form factor is now much more involved and I want to give some additional details concerning the approximations to be done.

The spinor matrix elements is obtained without any approximation :

$$
\begin{array}{r}
\overline{u_{\alpha}}\left(\mathbf{p}^{\prime}, s^{\prime}\right) \boldsymbol{\Gamma}_{\alpha} u_{\alpha}(\mathbf{p}, s)=\frac{\sqrt{\left(E_{p^{\prime}}+m\right)\left(E_{p}+m\right)}}{2 m} \\
\left\{\left(F_{1}\left(q^{2}\right)+\kappa F_{2}\left(q^{2}\right)\right)\right. \\
\left.\delta_{s^{\prime} s}\left(\frac{\mathbf{p}^{\prime}}{E_{p^{\prime}}+m}+\frac{\mathbf{p}}{E_{p}+m}\right)+i(\sigma)_{s^{\prime} s} \times\left(\frac{\mathbf{p}^{\prime}}{E_{p^{\prime}}+m}-\frac{\mathbf{p}}{E_{p}+m}\right)\right] \\
-\frac{\kappa}{2 m} F_{2}\left(q^{2}\right)\left(\mathbf{p}^{\prime}+\mathbf{p}\right)
\end{array}
$$




$$
\left.\left[\delta_{s^{\prime} s}\left(1-\frac{\mathbf{p}^{\prime} \cdot \mathbf{p}}{\left(E_{p^{\prime}}+m\right)\left(E_{p}+m\right)}\right)-i(\sigma)_{s^{\prime} s} \frac{\mathbf{p}^{\prime} \dot{\times} \mathbf{p}}{\left(E_{p^{\prime}}+m\right)\left(E_{p}+m\right)}\right]\right\}
$$

and a similar expression for the antiquark part of the current. In the right hand part of this equation all quantities must be understood with a label $\alpha$ $\left(E_{p}(\alpha), \kappa_{\alpha}, F_{1}^{\alpha}, \ldots\right)$. As in the NR case and for the same reason, the contribution of the spin dependent term vanishes. Due to the Wick contraction appearing in the expression of the current matrix element, the $\mathbf{p}$ and $\mathbf{p}^{\prime}$ variables of (10), should be understood as $\mathbf{p}_{1}$ and $\mathbf{p}_{1}^{\prime}$ (quark momenta in the meson). Denoting $\mathbf{S}=\mathbf{K}^{\prime}+\mathbf{K}$, and $\mathbf{Q}=\mathbf{K}^{\prime}-\mathbf{K}$, one has explicitly $\mathbf{p}_{1}=\mathbf{p}+\left(c_{\beta} / 2\right)(\mathbf{Q}-\mathbf{S})$ (here $\mathbf{p}$ is the relative momentum of the particles in the meson) and $\mathbf{p}_{1}^{\prime}=\mathbf{p}_{1}+\mathbf{Q}$. Instead, it is better to introduce the variable $\mathbf{q}=\left(\mathbf{p}_{1}+\mathbf{p}_{1}^{\prime}\right) / 2=\mathbf{p}+\left(c_{\alpha} / 2\right) \mathbf{Q}-$ $\left(c_{\beta} / 2\right) \mathbf{S}$ so that $\mathbf{p}_{1}=\mathbf{q}-\mathbf{Q} / 2$ and $\mathbf{p}_{1}^{\prime}=\mathbf{q}+\mathbf{Q} / 2$. The first approximation that I make is to suppose that $\mathbf{Q}$ is negligeable as compared to $\mathbf{q}$. Since the expression to calculate is symmetric in $\mathbf{p}_{1}$ and $\mathbf{p}_{1}^{\prime}$, this approximation is equivalent to $\mathbf{p}_{1}^{\prime}=\mathbf{p}_{1}=\mathbf{q}$ up to second order in $\mathbf{Q}$. Now one has a much simpler expression :

$$
\left\langle\Psi_{\mathbf{K}^{\prime}}\left|\mathbf{j}_{q}(0)\right| \Psi_{\mathbf{K}}\right\rangle=e_{\alpha} F_{1}^{\alpha}\left(-\mathbf{Q}^{2}\right) \int d \mathbf{p} \frac{\Psi\left(\mathbf{p}+c_{\alpha} \mathbf{Q}\right) \Psi(\mathbf{p})}{E_{q}(\alpha)} \mathbf{q}
$$

Up to now, this equation is exact to second order in $\mathbf{Q}$, but the integral is still too complicated to be calculated exactly. It is suitable to introduce the new variable $\mathbf{y}=\mathbf{p}+\left(c_{\alpha} / 2\right) \mathbf{Q}$; the last approximation that I make is to suppose $S<<y$. One has :

$$
\begin{gathered}
\left\langle\Psi_{\mathbf{K}^{\prime}}\left|\mathbf{j}_{q}(0)\right| \Psi_{\mathbf{K}}\right\rangle=e_{\alpha} F_{1}^{\alpha}\left(-\mathbf{Q}^{2}\right) \int d \mathbf{y} \frac{\Psi\left(\mathbf{y}+\left(c_{\alpha} / 2\right) \mathbf{Q}\right) \Psi\left(\mathbf{y}-\left(c_{\alpha} / 2\right) \mathbf{Q}\right)}{E_{y}(\alpha)} \\
\times\left[\mathbf{y}-\left(c_{\beta} / 2\right) \mathbf{S}\right]
\end{gathered}
$$

The new integral is composed of 2 terms. It is easy to show that the first term (proportional to $\mathbf{y}$ ) vanishes. The second term, proportional to $\mathbf{S}$, is just what one needs to make the identification giving the meson form factor. Doing that, one gets the final expression :

$$
F_{M}\left(\mathbf{Q}^{2}\right)=\frac{e_{\alpha}+e_{\beta}}{\widetilde{e}_{\alpha}+\widetilde{e}_{\beta}}\left[\widetilde{e}_{\alpha} F_{1}^{\alpha}\left(-\mathbf{Q}^{2}\right) J^{\alpha}\left(\mathbf{Q}^{2}\right)+\widetilde{e}_{\beta} F_{1}^{\beta}\left(-\mathbf{Q}^{2}\right) J^{\beta}\left(\mathbf{Q}^{2}\right)\right]
$$

where the dynamical integrals $J^{\rho}$ are defined by :

$$
J^{\rho}\left(\mathbf{Q}^{2}\right)=\widetilde{m}_{\rho} \int d \mathbf{y} \frac{\Psi\left(\mathbf{y}-\left(c_{\rho} / 2\right) \mathbf{Q}\right) \Psi\left(\mathbf{y}+\left(c_{\rho} / 2\right) \mathbf{Q}\right)}{E_{y}(\rho)} ; \rho=\alpha, \beta
$$


with the property $J^{\rho}(0)=1$. The remarkable fact concerning the "relativized meson form factor" (27), as its non relativistic version, is that it depends only on the $F_{1}$ quark form factor. Thus the quark anomalous magnetic moment cannot be determined from the meson form factor.

Concerning the charge square radius, the treatment is very similar to that of the NR case. Let us denote $K^{\rho}=-\left.6 \frac{d J^{\rho}\left(Q^{2}\right)}{d Q^{2}}\right|_{Q^{2}=0}$, the charge square radius can be written :

$$
\left\langle r_{R}^{2}\right\rangle_{M}=\left\langle r_{R}^{2}\right\rangle_{M}^{\text {bare }}+\frac{e_{\alpha}+e_{\beta}}{\widetilde{e}_{\alpha}+\widetilde{e}_{\beta}}\left[\widetilde{e}_{\alpha} G^{\alpha}+\widetilde{e}_{\beta} G^{\beta}\right]
$$

where the bare charge square radius, corrected by relativistic effects, is simply given by :

$$
\left\langle r_{R}^{2}\right\rangle_{M}^{\text {bare }}=\frac{e_{\alpha}+e_{\beta}}{\widetilde{e}_{\alpha}+\widetilde{e}_{\beta}}\left[\widetilde{e}_{\alpha} K^{\alpha}+\widetilde{e}_{\beta} K^{\beta}\right]
$$

Here again, the effect of a quark form factor is to enhance the meson square radius.

\section{$5 \quad$ Leptonic decays}

The decay of a vector meson into a lepton-antilepton pair $V \rightarrow l \bar{l}$ is governed essentially by creation and annihilation of a virtual photon. This is a perturbative process of second order in the hamiltonian $H_{e m}$ (11). The elementary amplitude, for which both the quarks and the leptons have definite momenta, can be calculated with the usual Feynman rules. The only difference concerns the quark-photon vertex where the term $\gamma^{\mu}$ should be replaced by $\Gamma^{\mu}\left(q^{2}\right)$. Moreover the initial state is a meson bound state and the elementary amplitude should be weighted with the probability that, in the meson, the quarks have a definite set of momenta (but this is precisely the wave function in momentum representation). Thus the starting point is the quantum amplitude of the process :

$$
\begin{aligned}
S_{V \rightarrow l \bar{l}}= & (2 \pi)^{4} \delta\left(\mathbf{P}_{1}+\mathbf{P}_{2}\right) \delta\left(2 E_{P}-M_{V}\right) \frac{m_{l}}{(2 \pi)^{3} E_{P}(l)} \\
& \times \sum_{\alpha_{1}, \alpha_{2}}\left\langle\alpha \alpha_{1} \alpha \alpha_{2}|| J M\right\rangle(-1)^{1 / 2-s_{2}} \\
& \times \int d \mathbf{p} \Psi(\mathbf{p}) \frac{m_{\alpha}}{(2 \pi)^{3} E_{p}(\alpha)} M\left(\mathbf{P}, \mathbf{p}, s_{1}, s_{2}\right)
\end{aligned}
$$


where the quantity $M$ is more or less the elementary amplitude defined here by :

$$
\begin{aligned}
M\left(\mathbf{P}, \mathbf{p}, s_{1}, s_{2}\right)=-i e^{2} e_{\alpha} \frac{g_{\mu \nu}}{q^{2}} & {\left[\overline{u_{l}}\left(\mathbf{P}, S_{1}\right) \gamma^{\mu} v_{l}\left(-\mathbf{P}, S_{2}\right)\right] } \\
\times & {\left[\overline{v_{\alpha}}\left(-\mathbf{p}, s_{2}\right) \Gamma_{\alpha}^{\nu}\left(q^{2}\right) u_{\alpha}\left(\mathbf{p}, s_{1}\right)\right] }
\end{aligned}
$$

The lepton (antilepton) momentum and spin polarization are denoted $\left(\mathbf{P}_{1}, S_{1}\right)$ (respectively $\left(\mathbf{P}_{2}, S_{2}\right)$ ) and their common flavour $l$. In the same spirit the quark (antiquark) momentum and spin polarization are denoted $\left(\mathbf{p}_{1}, s_{1}\right)$ (respectively $\left.\left(\mathbf{p}_{2}, s_{2}\right)\right)$ and their common flavour $\alpha$. I consider the decay in the center of mass frame and $\mathrm{I}$ have taken this condition by imposing $\mathbf{P}=\mathbf{P}_{1}=-\mathbf{P}_{2}$ and $\mathbf{p}=\mathbf{p}_{1}=-\mathbf{p}_{2}$.

In this case one has $q=-\left(p_{1}+p_{2}\right)=\left(-M_{V}, \mathbf{0}\right)$; we are in the time-like region and one must understand $q^{2}=M_{V}^{2}$ both in the photon propagator $\frac{g_{\mu \nu}}{q^{2}}$ and in the quark-photon vertex $\Gamma_{\alpha}^{\nu}\left(q^{2}\right)$. The usual approximation that is done is called van Royen-Weisskopf (VR) formalism [16]. It consists essentially to a NR reduction of spinors and neglecting the quark momenta distribution inside the mesons. The dynamical quantity that appears naturally is the wave function at the origin (in coordinate representation this time) : $\Psi_{V}(0)$. An immediate consequence is that the decay occurs only for ${ }^{3} S_{1}$ resonances $(L=0)$. In fact, the calculation of (31-32) can be performed without any approximation. I will not enter into details because the philosophy is given in [17]. I just want to say some words on the novelty of this paper, namely the presence of the quark form factor. The basic quantity is $W_{\alpha}^{\nu}=\overline{v_{\alpha}}\left(-\mathbf{p}, s_{2}\right) \Gamma_{\alpha}^{\nu}\left(M_{V}^{2}\right) u_{\alpha}\left(\mathbf{p}, s_{1}\right)$. Using again the Gordon identity, this term is a sum of two contributions :

$$
W_{\alpha}^{\nu}=A_{\alpha} X_{\alpha}^{\nu}+B_{\alpha} Y_{\alpha}^{\nu}
$$

where

$$
A_{\alpha}=F_{1}^{\alpha}\left(M_{V}^{2}\right)+\kappa_{\alpha} F_{2}^{\alpha}\left(M_{V}^{2}\right) ; B_{\alpha}=\frac{\kappa_{\alpha}}{2 m_{\alpha}} F_{2}^{\alpha}\left(M_{V}^{2}\right)
$$

One has also $W_{\alpha}^{0}=0$ and for the corresponding $\mathbf{X}_{\alpha}$ and $\mathbf{Y}_{\alpha}$ quantities:

$$
\mathbf{X}_{\alpha}=\overline{v_{\alpha}}\left(-\mathbf{p}, s_{2}\right) \gamma u_{\alpha}\left(\mathbf{p}, s_{1}\right)=\frac{E_{p}(\alpha)}{m_{\alpha}}(\sigma)_{-s_{2}, s_{1}}-\mathbf{p} \frac{(\sigma \cdot \mathbf{p})_{-s_{2}, s_{1}}}{m_{\alpha}\left(E_{p}(\alpha)+m_{\alpha}\right)}
$$

and

$$
\mathbf{Y}_{\alpha}=-2 \overline{v_{\alpha}}\left(-\mathbf{p}, s_{2}\right) \mathbf{p} u_{\alpha}\left(\mathbf{p}, s_{1}\right)=\frac{2}{m_{\alpha}} \mathbf{p}(\sigma \cdot \mathbf{p})_{-s_{2}, s_{1}}
$$


The net result of the complete calculation is just the replacement of $\Psi_{V}(0)$ by a modified quantity :

$$
\widetilde{\Psi}_{V}(0)=\delta_{L, 0} A_{\alpha} \Psi_{V}(0)+\frac{\sqrt{2(2 L+1)}}{6 \pi}\left\langle\begin{array}{lllllllllll}
0 & 1 & 0 & 0 & 1 & 0 & I_{V}^{\alpha}
\end{array}\right.
$$

In addition to $\Psi_{V}(0)$, the meson wave function also participates to the dynamical integral :

$$
I_{V}^{\alpha}=\int_{0}^{\infty} d p p^{4} T_{\alpha}(p) \varphi_{V}(p)
$$

where

$$
T_{\alpha}(p)=\frac{2 B_{\alpha}}{E_{p}(\alpha)}-\frac{A_{\alpha}}{E_{p}(\alpha)\left[E_{p}(\alpha)+m_{\alpha}\right]}
$$

Dealing with point-like quarks corresponds to the condition $A_{\alpha}=1$ and $B_{\alpha}=$ 0 in (37). Even in this case, the formalism differs from the VR prescription, because of the presence of the dynamical integral ( 38); this last quantity takes care of two phenomena : the momentum distribution of the quarks inside the meson through the $\varphi_{V}(p)$ factor and a relativistic correction through the $E_{p}(\alpha)$ terms in the $T_{\alpha}(p)$ factor. A consequence of (37) is that the decay can occur not only from ${ }^{3} S_{1}$ resonance (with a modified amplitude) but also from ${ }^{3} D_{1}$ resonance.

Up to now, I have supposed one colour and one flavour to write the amplitude; this amplitude does not depend on colour and depends on flavour explicitly through the charge $e_{\alpha}$ and implicitly through the wave function $\varphi_{V}(p)$. Taking into account colour degrees of freedom gives a multiplicative factor 3 in the width as compared to the original VR formula (at that time the authors were not aware about colour). Taking into account the mixing of flavour (the $\rho^{0}$ is a mixing of $u \bar{u}$ and $d \bar{d}$ ) means the replacement of $e_{\alpha} \widetilde{\Psi}_{V}(0)$ by the summation over all the flavours $f$ entering in the composition of the meson, with the corresponding probability amplitudes $c_{f}: \sum_{f} c_{f} e_{f} \widetilde{\Psi}_{V}^{f}(0)$.

The rest of the calculation is long but presents no difficulty. The final expression for the leptonic width is given by ( $\alpha$ is the fine structure constant):

$$
\Gamma(V \rightarrow \bar{l})=\delta_{S, 1} \delta_{J, 1} 16 \pi \frac{\alpha^{2}}{M_{V}^{2}}\left(1+\frac{2 m_{l}^{2}}{M_{V}^{2}}\right)\left(1-\frac{4 m_{l}^{2}}{M_{V}^{2}}\right)^{1 / 2}
$$




$$
\times\left(\sum_{f} c_{f} e_{f} \widetilde{\Psi}_{V}^{f}(0)\right)^{2}
$$

In all my calculations, I apply this exact formula but, most of the time, the terms $m_{l}^{2} / M_{V}^{2}$ are negligeable, so that, practically, the decay does not depend upon the lepton flavour in the final channel.

\section{Results}

\subsection{Strategy}

As stated in the introduction, I consider in this paper two different types of quark-antiquark potentials : DNR to be used with a NR kinematical energy, and DSR to be used with a R kinematical energy [5]. Once the corresponding wave functions are obtained by solving the Schrödinger or the Salpeter equation, the bare quantities, as well as the quantities modified by relativistic corrections, are completely determined without any free parameter. It is convenient to expand the wave function in momentum representation as a sum of gaussian terms; all these quantities can be computed rather easily, as it is shown in the appendix. In practice, I restrict myself to 5 gaussian terms; this is an excellent approximation for the exact wave function.

Concerning the meson form factor, it depends on a free function $F_{1}\left(q^{2}\right)$. The dipole form $F_{1}\left(q^{2}\right)=1 /\left(1-q^{2} / \Lambda^{2}\right)^{2}$ is the traditional prescription used for the nucleon form factor [10]-[11]; since there are some similarities between quarks and nucleons, such a form seems reasonable. But the asymptotic behaviour of meson form factors is believed [15] to be $Q^{-2}$ so that a monopole form $F_{1}\left(q^{2}\right)=1 /\left(1-q^{2} / \Lambda^{2}\right)$ seems more appropriate. In this paper I suggest to test both forms.

For the meson form factor, the expression in the space-like region is needed; thus the bare quantities are more or less renomalized by the function $F_{1}\left(-\mathrm{Q}^{2}\right)=$ $1 /\left(1+\mathbf{Q}^{2} / \Lambda^{2}\right)^{n}$. I suppose here that $F_{1}^{u}=F_{1}^{d}=F_{1}^{n}$, as suggested by isospin invariance. Thus the pion form factor depends on only one free parameter: the cut off for the normal quark $\Lambda_{n}=\Lambda_{u}=\Lambda_{d}$. I determine it from a best fit on the experimental data. The kaon form factor depends on $F_{1}^{n}$ and $F_{1}^{s}$. I keep the value of $\Lambda_{n}$ as found previously, and determine $\Lambda_{s}$ from a best fit of the data. Thus the quark form factor can be obtained quite easily from the meson form factor in the ordinary and strange sector. It is a pity that no data exist in the charm and bottom sectors, so that $\Lambda_{c}$ and $\Lambda_{b}$ remain free parameters for the moment. 


\begin{tabular}{|r|r|r|r|r|}
\hline & DNR-M form & DNR-D form & DSR-M form & DSR-D form \\
\hline$\Lambda_{u}=\Lambda_{d}$ & 0.953 & 1.492 & 0.8035 & 1.294 \\
\hline$\Lambda_{s}$ & 1.437 & 1.912 & 1.3095 & 1.705 \\
\hline$\Lambda_{c}$ & 15.3 & 30.0 & 10.0 & 30.0 \\
\hline$\Lambda_{b}$ & 40.0 & 55.0 & 40.0 & 60.0 \\
\hline$\kappa_{u}$ & -0.488 & -0.368 & -0.5125 & -0.344 \\
\hline$\kappa_{d}$ & -0.466 & -0.337 & -0.5225 & -0.329 \\
\hline$\kappa_{s}$ & -0.513 & -0.504 & -0.504 & -0.496 \\
\hline$\kappa_{c}$ & -0.183 & -0.150 & -0.316 & -0.260 \\
\hline$\kappa_{b}$ & -0.055 & -0.055 & -0.160 & -0.150 \\
\hline
\end{tabular}

Table 1

Parameters for the electromagnetic quark form factors. Two forms are studied : monopole form ( $\mathrm{M}$ form) and dipole form ( $\mathrm{D}$ form). Two different quark -antiquark potentials are considered : DNR and DSR. For the determination of the parameters, see text.

I have no real argument to choose a definite form for $F_{2}\left(q^{2}\right)$; by simplicity I take $F_{2}\left(q^{2}\right)=F_{1}\left(q^{2}\right)$. The only parameters that remain to be determined are the quark anomalous magnetic moments. To proceed further, I consider the $\rho^{0} \rightarrow e^{+} e^{-}$and $\omega \rightarrow e^{+} e^{-}$decays; they allow to fix the $\kappa_{u}$ and $\kappa_{d}$ values. The $\phi \rightarrow e^{+} e^{-}$decay provides the $\kappa_{s}$ parameter. In the charmonium sector a number of leptonic decays are known experimentally; I use them to get $\Lambda_{c}$ and $\kappa_{c}$. In the same manner, the bottomium sector provides me with $\Lambda_{b}$ and $\kappa_{b}$. Let us remark that, for leptonic decays, the time-like region of the quark form factors is explored : $F_{1}\left(M_{V}^{2}\right)=1 /\left(1-M_{V}^{2} / \Lambda^{2}\right)^{n}$; if the resonance has a mass that is close to the cut off, one may have troubles.

The strategy developed just above allows a complete determination of all free parameters. Moreover there exist more experimental data than free parameters so that the theory can be checked correctly. All the quark electromagnetic parameters are summarized in Table 1 for both potentials DNR and DSR and for both forms of $F_{2}\left(q^{2}\right)=F_{1}\left(q^{2}\right)$.

Some comments are in order. The meson form factors are sensitive to the cut off, which are thus constrained rather seriously. Due to the experimental situation, only $\Lambda_{n}$ and $\Lambda_{s}$ are obtained precisely. On the contrary, the very influential parameter for the leptonic decays is the quark anomalous magnetic moment; the cut off does not play an important role and could be change by $50 \%$ without affecting too much the results. Thus the values for $\Lambda_{c}$ and $\Lambda_{b}$ given in the table 1 are just reasonable values, that can be varied a bit without dramatic consequences. 
The cut off obtained with a monopole form are systematically lower than those obtained with a dipole form. This is the consequence of a slower asymptotic behaviour. The values are around 2-3 times the corresponding quark mass for the light sector and around 8-10 times the quark mass for the heavy sector. This is due to the fact that dynamical relativistic effects are weaker in the heavy sector with the consequence that a non relativistic expression for the wave function is closer to reality. With a monopole form, that is in principle more suited for describing the behaviour at large $Q^{2}$, the value for $\Lambda_{n}$ is of order of the mass of the $\rho$ resonance. Although this parameter was obtained in a purely phenomenological way, it corresponds more or less to what is expected from the vector dominance model (VDM). Indeed, a possible explanation of the $F_{1}\left(Q^{2}\right)$ term at the quark-photon vertex could be a coupling of a $u$ quark to the $\rho$ resonance with a subsequent decay of this resonance into a photon. This is the essence of VDM and, in this frame, a correction factor proportional to the $\rho$ propagator is expected : this would result in a term $m_{\rho}^{2} /\left(m_{\rho}^{2}+Q^{2}\right)$ with the consequence that $\Lambda_{u}=\Lambda_{d}=m_{\rho}$. Within this model, the kaon form factor requires the propagation of a $\phi$ from the $\bar{s}$ antiquark line. This would imply $\Lambda_{s}=m_{\phi}=1020 \mathrm{MeV}$. Again, the fitted value is not so far from this last value. One must also keep in mind that the origin of the $F_{1}\left(Q^{2}\right)$ function is not entirely due to VDM.

All the anomalous moments should be taken negative. I have no explanation for that. The value $\kappa_{u}$ is very close to $\kappa_{d}$. I see no reason for a strict equality, but again I do not find any explanation for that phenomenon. Their values in the heavy sector are smaller; this is related to the above mentioned property of the wave function. Do not forget that a point-like quark corresponds to the limit $\kappa=0, \Lambda=\infty$. From purely kinematical arguments, I would expect that the magnetic moment for the $s$ quark is weaker than the one corresponding to the ordinary quark. In fact both quantities appear to have the same order of magnitude. The leptonic widths are very sensitive to this parameter and requiring that $\left|\kappa_{s}\right|$ is appreciably smaller than $\left|\kappa_{u}\right|$ would spoil the description of the $\phi$ decay. I have no satisfactory explanation for that feature at the moment.

\subsection{Charge mean square radius}

In this section, I study the meson charge mean square radii of the pion and the kaon in the formalism developed previously for the wave function resulting from the DNR and DSR potentials. The bare non-relativistic approximation is given by (21), and calculated practically by (42); it is denoted bareNR. The bare relativistic approximation is given by (30), and calculated practically by (45); it is denoted bareR. The theoretical values of both approximations are obtained without free parameter; they are the consequence of the internal 
Table 2

\begin{tabular}{|r|r|r|}
\hline & $\pi$ & $K$ \\
\hline $\exp \left(\mathrm{GeV}^{-2}\right)$ & 10.97 & 8.73 \\
\hline DNR-bareNR & 2.35 & 2.64 \\
\hline DNR-bareR & 2.49 & 2.68 \\
\hline DNR-M form & 9.10 & 7.85 \\
\hline DNR-D form & 7.89 & 7.25 \\
\hline DSR-bareNR & 1.05 & 1.52 \\
\hline DSR-bareR & 1.33 & 1.69 \\
\hline DSR-M form & 10.62 & 8.46 \\
\hline DSR-D form & 8.50 & 7.53 \\
\hline
\end{tabular}

Values of the pion and kaon charge mean square radii. The experimental data are reported in the first line. Two different quark- antiquark potentials are considered : DNR and DSR. Four treatments are studied : non-relativistic and no quark form factor (bareNR), relativistic and no quark form factor (bareR), relativistic plus a monopole quark form factor (M form), relativistic plus a dipole quark form factor (D form). All values are given in $\mathrm{GeV}^{-2}$.

quark dynamics inside mesons. The most sophisticated treatment includes relativistic corrections and quark form factors; the charge square radius is given by (29). Both forms are studied; the results for the monopole form are denoted M-form and for them $G^{\rho}=6 / \Lambda_{\rho}^{2}$; those for the dipole form are denoted D-form and for them $G^{\rho}=12 / \Lambda_{\rho}^{2}$. All these results are presented in Table 2.

The bare approximations largely underestimate the values; the relativistic corrections are not very important but always go in the right direction. The M-form is clearly better than D-form for both potentials. Although the bare approximations are much poorer for DSR than for DNR, the values corrected with the quark form factor appear a little better.

\subsection{Meson form factor}

The NR expression for the form factor is given by (17) and calculated practically with formula (46) and the $\mathrm{R}$ expression by (27) and calculated with formula (48). The case of point-like quarks, called bare form factors, is very easy to obtain : it corresponds to $F_{1}\left(-\mathrm{Q}^{2}\right)=1$ in those formulae.

To have an idea of the modification of the quark masses and charges due to relativistic corrections, I present on table 3 the constituents masses $m_{\alpha}$, the 


\section{$\pi$ form factor}

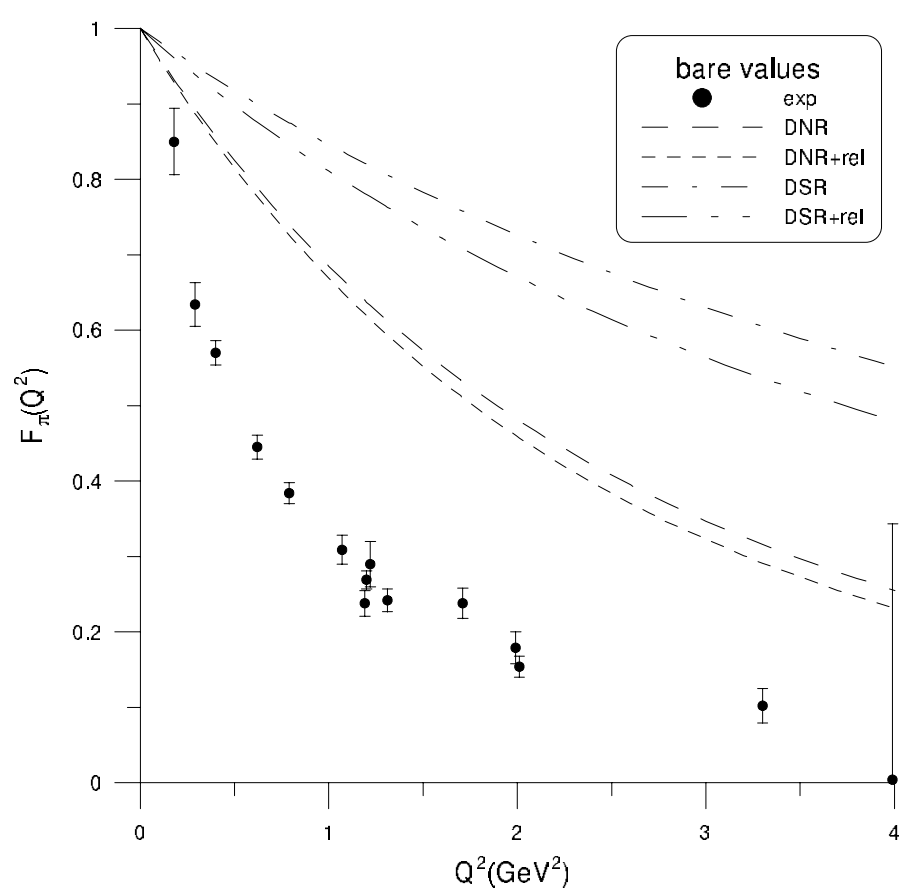

Fig. 1. Pion bare form factor. The experimental data (filled circles) and their error bars come from [18]. The form factors calculated with DNR wave function are plotted with dashed lines (long for NR, short for R). The form factors calculated with DSR wave function are plotted with dash-dot lines (1 dot for NR, 2 dots for $\mathrm{R})$.

renormalized masses $\widetilde{m}_{\alpha}$ (see equation (22)) and the renormalized charges $\widetilde{e}_{\alpha}$ (see equation (23)) for the quarks of the light sector, obtained with DNR and DSR wave functions. The $\widetilde{m}_{\alpha}$ quantities are always larger than the corresponding $m_{\alpha}$, and consequently $\widetilde{e}_{\alpha}$ are smaller than the bare $e_{\alpha}\left(e_{u}=2 / 3, e_{\bar{d}}=1 / 3\right.$ $\left.=e_{\bar{s}}\right)$.

\section{Table 3}

\begin{tabular}{|r|r|r|r|}
\hline & $u$ & $\bar{d}$ & $\bar{s}$ \\
\hline$m_{\alpha}(\mathrm{DNR})$ & 0.3500 & 0.3500 & 0.6222 \\
\hline$\widetilde{m}_{\alpha}(\mathrm{DNR})$ & 0.5447 & 0.5447 & 0.7830 \\
\hline$\widetilde{e}_{\alpha}(\mathrm{DNR})$ & 0.4284 & 0.2142 & 0.2649 \\
\hline$m_{\alpha}(\mathrm{DSR})$ & 0.2210 & 0.2210 & 0.4340 \\
\hline$\widetilde{m}_{\alpha}(\mathrm{DSR})$ & 0.5988 & 0.5988 & 0.7366 \\
\hline$\widetilde{e}_{\alpha}(\mathrm{DSR})$ & 0.2461 & 0.1230 & 0.1964 \\
\hline
\end{tabular}

Constituent masses (in $\mathrm{GeV}$ ), renormalized masses (in $\mathrm{GeV}$ ) and renormalized charges (dimensionless) for the light quarks and for DNR wave functions (upper rows) and DSR wave functions (lower rows) 
The bare form factors for the pion are shown on fig 1 for both potentials and compared to the experimental data. The DNR wave function, although not so good, appears much better than DSR wave function. The relativistic corrections go in the right direction and are more sensitive for the DSR potential.

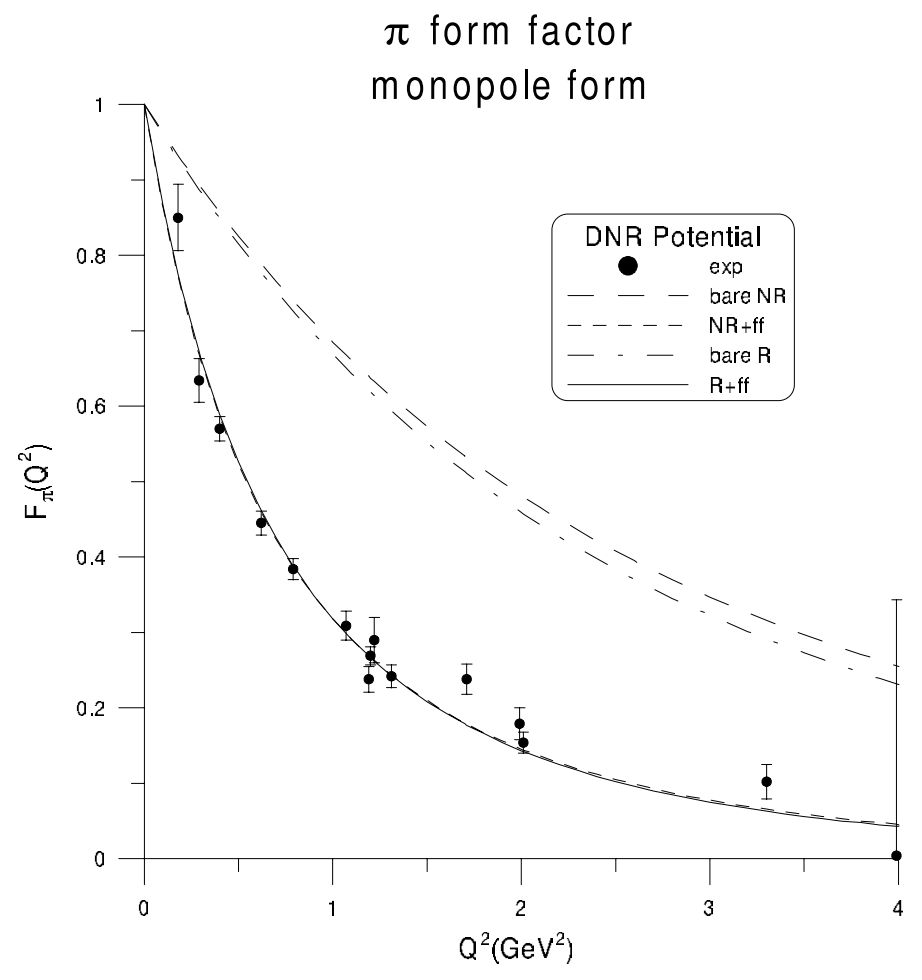

Fig. 2. Pion form factor obtained with DNR wave function and a monopole form for the quark form factor. Long dashed line corresponds to bare NR, dash-dot line to bare R, short dashed line to NR approximation with presence of a monopole quark form factor, and solid line to $\mathrm{R}$ approximation with a quark form factor.

In fig 2, I plot the pion form factor obtained with DNR wave function, a monopole form and for 4 different types of calculation : the bare NR expression, the bare $\mathrm{R}$ expression, the NR expression but with a quark form factor and the $\mathrm{R}$ expression with a quark form factor. In this last two cases, the cut off factor is fitted to the data. One sees that a good fit can be obtained in each case, at the price of a small change in the cut off value.

The similar plot for a dipole form is shown in fig 3 . The description is also correct but the behaviour for high momentum transfer is different and this case is a little poorer than the previous one.

In fig 4, I represent the results with a monopole form but obtained with a DSR wave function. One sees that the agreement is now excellent, even better than in fig 2. This means that, although the quark form factor is the dominant effect for the description of the meson form factor, the wave function (that comes from a QCD dynamics) also plays a role. 


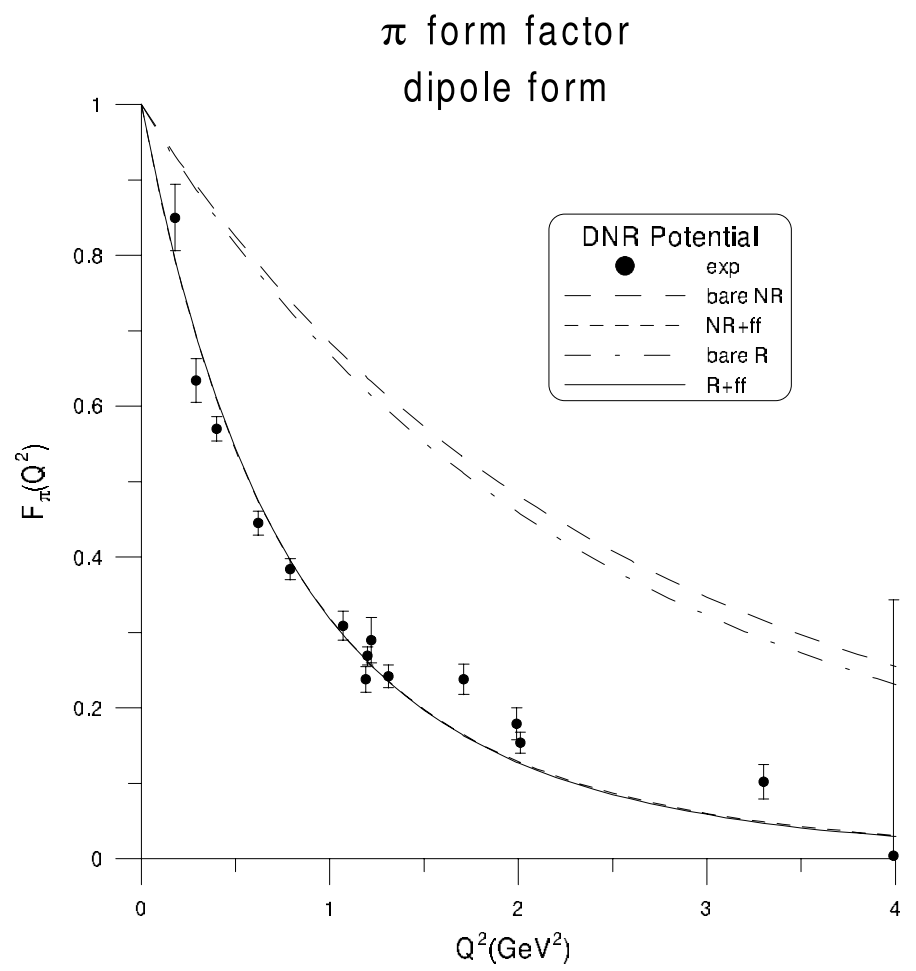

Fig. 3. Same as fig.2 for a dipole form.

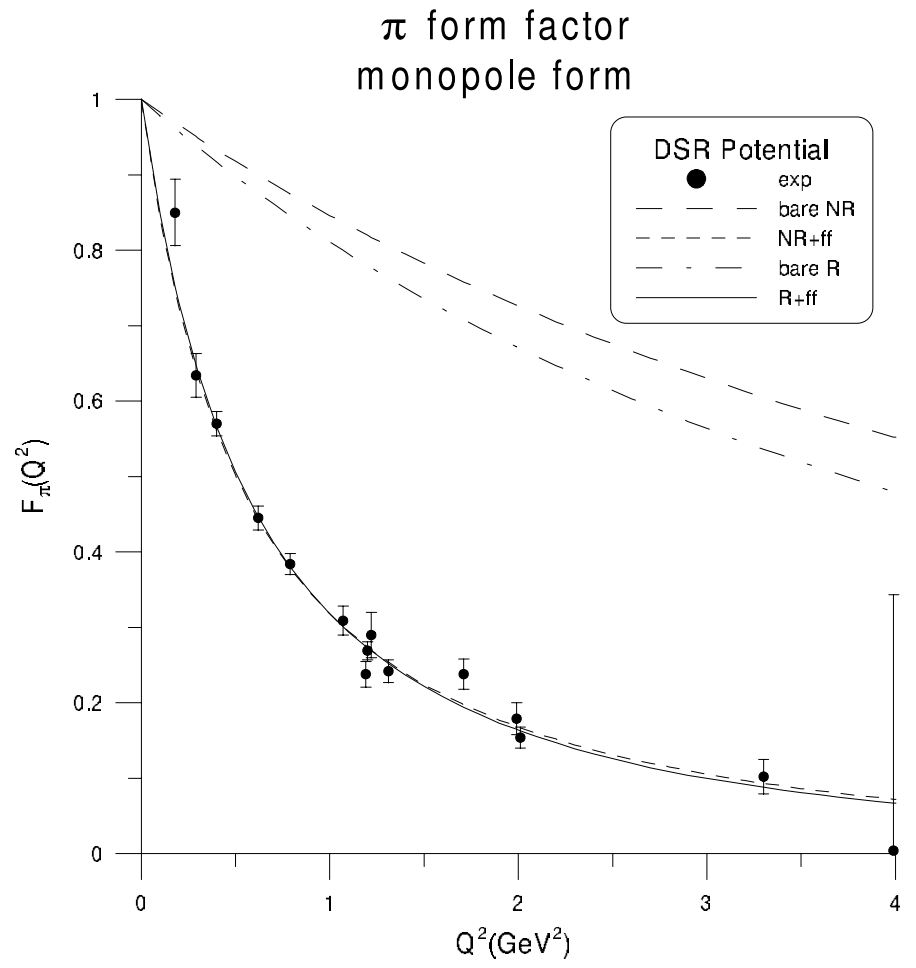

Fig. 4. Same as fig.2 for DSR wave function.

The data concerning the kaon form factor are less precise. To have a glance on what can be obtained in this case, I present in fig 5 the form factor obtained 


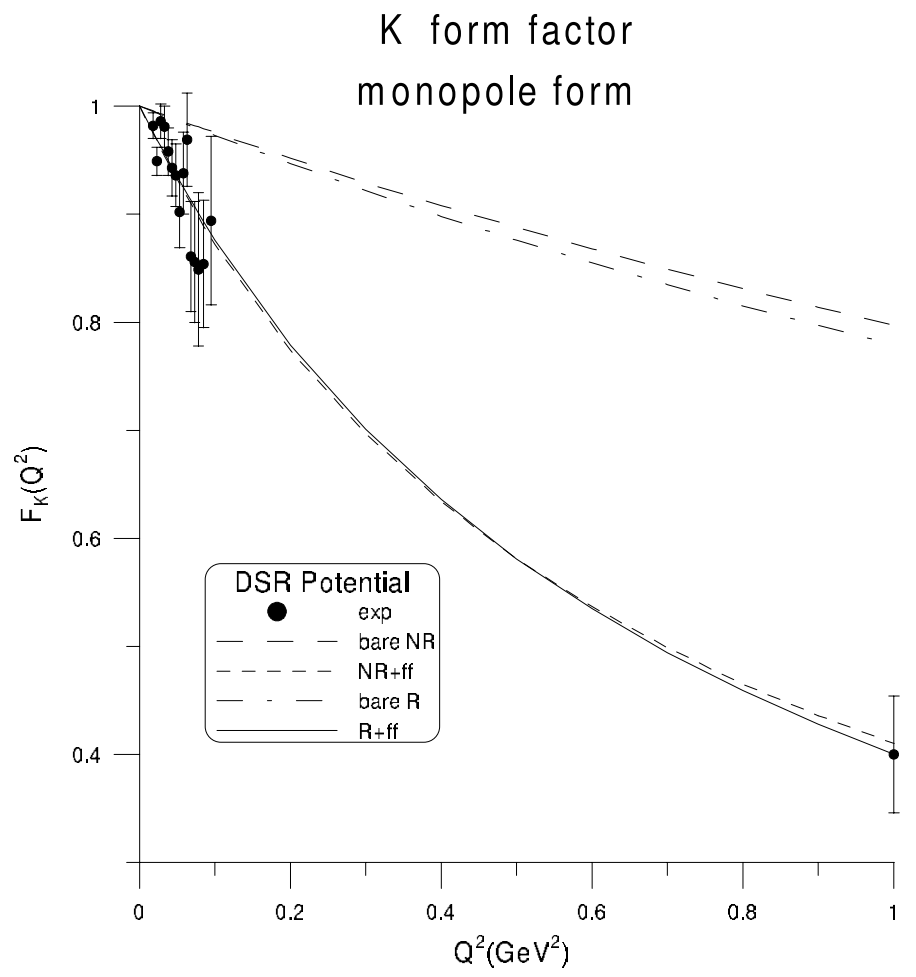

Fig. 5. Kaon form factor obtained with DSR wave function and a monopole form for the quark form factor. Same meaning as in fig.2. Experimental data for low momenta come from [19] and the isolated point, very useful, come from [20].

\begin{tabular}{|l|r|r|r|r|r|r|}
\hline & bare NR & bare R & NR-M form & NR-D form & R-M form & R-D form \\
\hline DNR & 344.69 & 311.05 & 1.677 & 3.190 & 1.827 & 3.354 \\
\hline DSR & 797.39 & 679.41 & 1.330 & 1.993 & 1.157 & 2.182 \\
\hline
\end{tabular}

Chi square values calculated on all known data for the pion form factor. See text for the definition of the chi square. The meaning of the entries are identical to those of table 2 .

with DSR potential and a monopole form. The results look quite good here too.

Just to give an idea of the quality of the fit, I report on Table 4 , the chi square $\chi^{2}=\frac{1}{N} \sum_{i=1}^{N}\left(f f^{t h}(i)-f f^{\exp }(i)\right)^{2} /$ error $^{\exp }(i)^{2}$ based on available data for the pion form factors. Various treatments are indicated. All the comments concerning the figures are quantitatively illustrated.

\subsection{Leptonic decays}

In this section, I study the leptonic decays of a vector meson with the two types of wave function. I focus my interest on 3 different treatments : the 


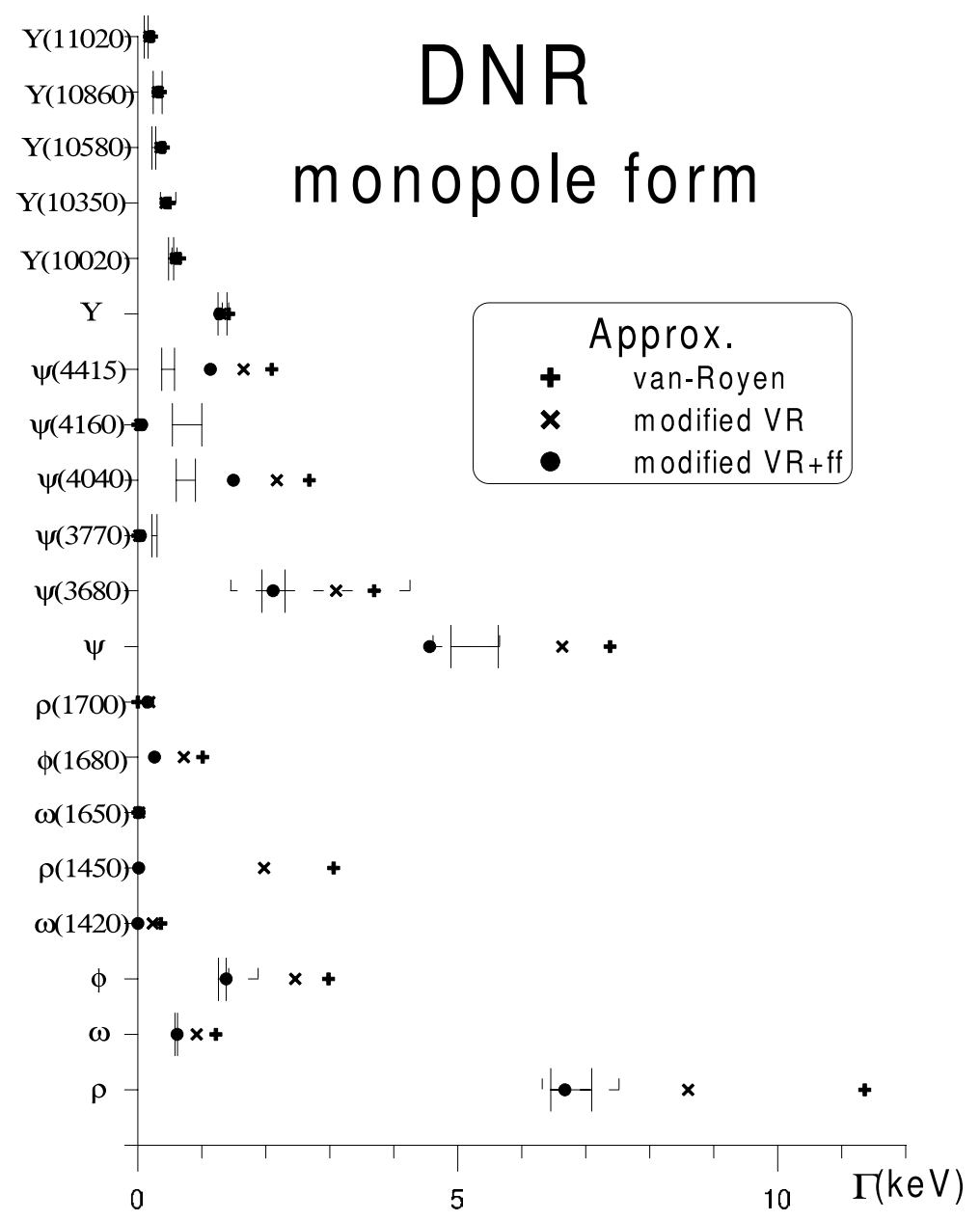

Fig. 6. Leptonic decay width (in $\mathrm{KeV}$ ) obtained with $\mathrm{DNR}$ wave function and a monopole form for quark form factor. The experimental values for $e^{+} e^{-}$decays are symbolized by solid error bars, and for $\mu^{+} \mu^{-}$by dashed error bars. They come from [21]. The theoretical values obtained with VR approximation are denoted with a + sign, the $\mathrm{m}-\mathrm{VR}$ with $\mathrm{a} \times$ sign, and the exact value with quark form factor with a circle.

van Royen (VR) approximation $\left(\widetilde{\Psi}_{V}(0)=\Psi_{V}(0)\right)$, the VR approximation with modification due to relativity and quark momentum distribution inside the meson $\left(\widetilde{\Psi}_{V}(0)\right.$ as in (37) with $A_{\alpha}=1$ and $\left.B_{\alpha}=0\right)(\mathrm{m}-\mathrm{VR})$, the total calculation corresponding to the $\mathrm{m}-\mathrm{VR}$ with contribution of quark form factor calculated with the parameters of table 1 .

Let me remind that the first two treatments are free from any parameter and that the third case essentially allows the determination of the quark anomalous magnetic moment. The decays of ${ }^{3} S_{1}$ resonances are allowed in every case, while the decays of ${ }^{3} D_{1}$ resonances are forbidden by VR. All other resonances are strictly forbidden in my formalism. I study all the known transitions in that framework. In fig 6, I present the results obtained with DNR potential and a monopole form. In the bottomium sector the VR approximation gives reason- 


\begin{tabular}{|c|c|c|c|c|}
\hline & VR & $\mathrm{m}-\mathrm{VR}$ & M-form & $\exp$ \\
\hline$\rho \rightarrow e^{+} e^{-}$ & 11.36 & 8.60 & 6.67 & $6.77 \pm 0.32$ \\
\hline$\rho \rightarrow \mu^{+} \mu^{-}$ & 11.33 & 8.58 & 6.65 & $6.92 \pm 0.60$ \\
\hline$\omega \rightarrow e^{+} e^{-}$ & 1.217 & 0.922 & 0.613 & $0.60 \pm 0.02$ \\
\hline$\omega \rightarrow \mu^{+} \mu^{-}$ & 1.215 & 0.920 & 0.612 & ¡ 1.5 \\
\hline$\phi \rightarrow e^{+} e^{-}$ & 2.984 & 2.464 & 1.375 & $1.32 \pm 0.06$ \\
\hline$\phi \rightarrow \mu^{+} \mu^{-}$ & 2.982 & 2.462 & 1.374 & $1.65 \pm 0.23$ \\
\hline$\omega(1420) \rightarrow e^{+} e^{-}$ & 0.363 & 0.233 & 0.0007 & seen \\
\hline$\rho(1450) \rightarrow e^{+} e^{-}$ & 3.063 & 1.970 & 0.016 & seen \\
\hline$\omega(1650) \rightarrow e^{+} e^{-}$ & 0 & 0.021 & 0.023 & seen \\
\hline$\phi(1680) \rightarrow e^{+} e^{-}$ & 1.010 & 0.722 & 0.259 & seen \\
\hline$\rho(1700) \rightarrow e^{+} e^{-}$ & 0 & 0.179 & 0.152 & seen \\
\hline$J / \psi \rightarrow e^{+} e^{-}$ & 7.381 & 6.634 & 4.561 & $5.26 \pm 0.37$ \\
\hline$J / \psi \rightarrow \mu^{+} \mu^{-}$ & 7.381 & 6.634 & 4.561 & $5.13 \pm 0.52$ \\
\hline$\psi(3680) \rightarrow e^{+} e^{-}$ & 3.696 & 3.101 & 2.106 & $2.12 \pm 0.18$ \\
\hline$\psi(3680) \rightarrow \mu^{+} \mu^{-}$ & 3.696 & 3.101 & 2.106 & $2.85 \pm 1.40$ \\
\hline$\psi(3770) \rightarrow e^{+} e^{-}$ & 0 & 0.023 & 0.040 & $0.26 \pm 0.04$ \\
\hline$\psi(4040) \rightarrow e^{+} e^{-}$ & 2.681 & 2.175 & 1.478 & $0.75 \pm 0.15$ \\
\hline$\psi(4160) \rightarrow e^{+} e^{-}$ & 0 & 0.032 & 0.059 & $0.77 \pm 0.23$ \\
\hline$\psi(4415) \rightarrow e^{+} e^{-}$ & 2.087 & 1.652 & 1.135 & $0.47 \pm 0.10$ \\
\hline$\Upsilon(9460) \rightarrow e^{+} e^{-}$ & 1.415 & 1.299 & 1.284 & $1.32 \pm 0.07$ \\
\hline$\Upsilon(9460) \rightarrow \mu^{+} \mu^{-}$ & 1.415 & 1.299 & 1.284 & $1.37 \pm 0.05$ \\
\hline$\Upsilon(9460) \rightarrow \tau^{+} \tau^{-}$ & 1.404 & 1.288 & 1.273 & $1.40 \pm 0.13$ \\
\hline$\Upsilon(10020) \rightarrow e^{+} e^{-}$ & 0.654 & 0.588 & 0.589 & $0.52 \pm 0.04$ \\
\hline$\Upsilon(10020) \rightarrow \mu^{+} \mu^{-}$ & 0.654 & 0.588 & 0.589 & $0.57 \pm 0.04$ \\
\hline$\Upsilon(10020) \rightarrow \tau^{+} \tau^{-}$ & 0.650 & 0.585 & 0.586 & $0.75 \pm 0.70$ \\
\hline$\Upsilon(10350) \rightarrow \mu^{+} \mu^{-}$ & 0.488 & 0.432 & 0.436 & $0.47 \pm 0.12$ \\
\hline$\Upsilon(10580) \rightarrow e^{+} e^{-}$ & 0.402 & 0.353 & 0.358 & $0.25 \pm 0.03$ \\
\hline$\Upsilon(10860) \rightarrow e^{+} e^{-}$ & 0.351 & 0.306 & 0.312 & $0.31 \pm 0.07$ \\
\hline$\Upsilon(11020) \rightarrow e^{+} e^{-}$ & 0.208 & 0.179 & 0.183 & $0.13 \pm 0.03$ \\
\hline
\end{tabular}

Table 5

Leptonic widths (in $\mathrm{KeV}$ ) for all known experimental decays of vector mesons. The 3 treatments Van-Royen (VR), modified Van-Royen (m-VR), exact with a monopole form (M-form) are detailed in the text. The values correspond to a DNR wave function. $\rho, \omega$ and $\phi$ decays give $\kappa_{u}, \kappa_{d}$ zand $\kappa_{s}$ while $\kappa_{c}, \Lambda_{c}\left(\kappa_{b}, \Lambda_{b}\right)$ are obtained from a fit on charmonium (bottomium) transitions. 
able values, while for other sectors it gives overestimated values, although the order of magnitude is correct. The effect of the modification is to reduce the VR values; thus the new results are in better agreement with experiment. If in addition I switch on the quark form factor, the theoretical values come very close to the experimental ones. Therefore, the overall description is strongly improved with the suggested sophistication. The corresponding quantitative values are summarized on Table 5 . I do not present the results with a dipole form, because they are of the same quality.

It is remarkable that, whatever the decay under consideration, the $\mathrm{m}-\mathrm{VR}$ approximation is significantly better than the original VR approximation, and that the presence of a quark form factor still improves strongly the results. In the light quark sector, the transitions that are "seen" have presumably a narrow width and this is precisely what is obtained. In the heavy quark sector the ${ }^{3} S_{1}$ decays are nicely reproduced, while the ${ }^{3} D_{1}$ decay widths (forbidden in VR) remain still too low.

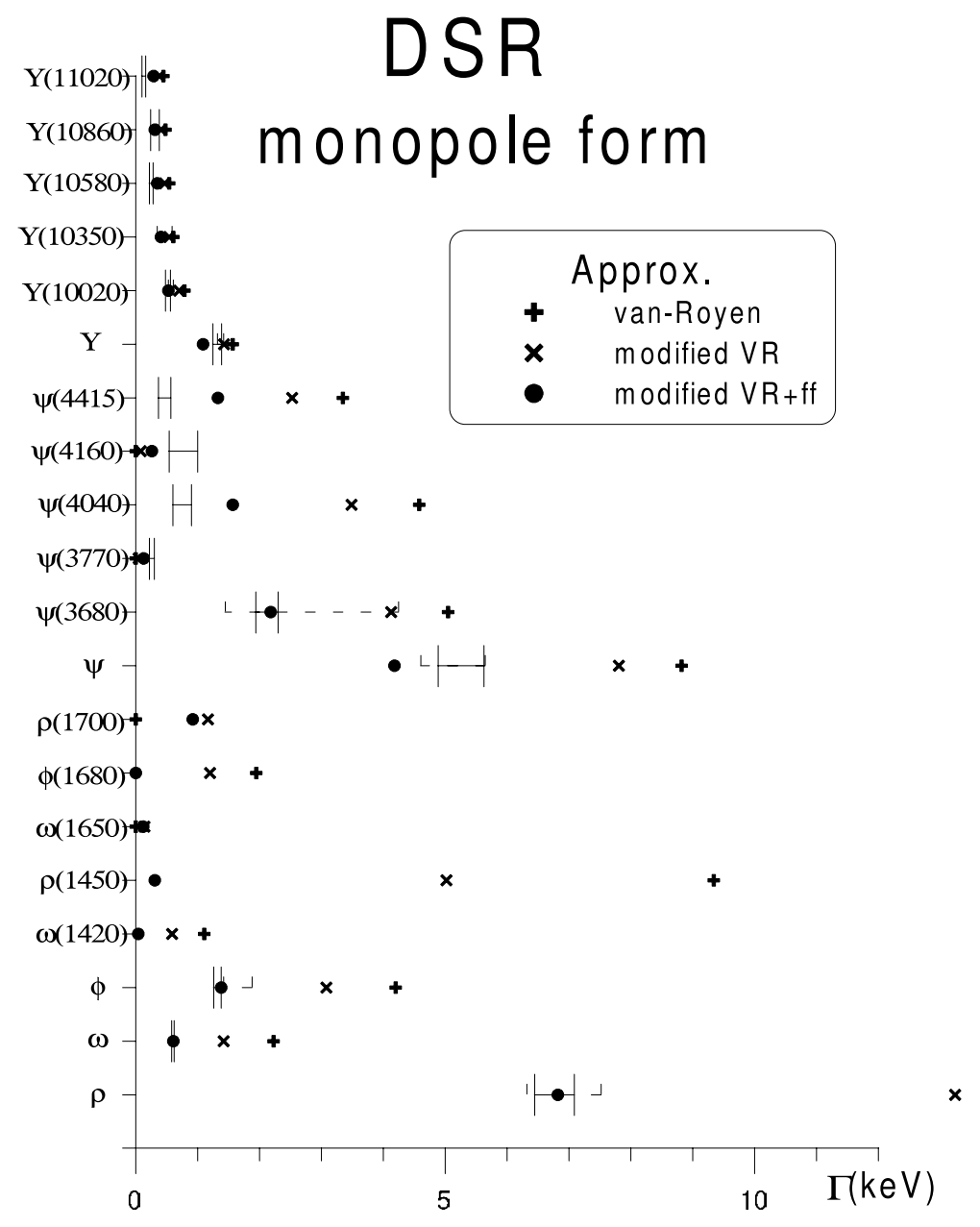

Fig. 7. Same as fig.6 for DSR wave function.

In fig 7 is plotted the situation for the DSR potential and a monopole form. 
Table 6

\begin{tabular}{|r|r|r|r|r|}
\hline & VR & m-VR & M-form & D-form \\
\hline DNR & 116.28 & 44.50 & 6.10 & 6.33 \\
\hline DSR & 597.40 & 184.70 & 9.71 & 8.86 \\
\hline
\end{tabular}

Chi square values calculated on all known leptonic decays. Two different wave functions are considered : DNR and DSR. Four treatments are studied : van Royen approximation (VR), modified van Royen (m-VR), complete calculation with a monopole (M-form) and a dipole (D-form) quark form factor.

The conclusion is essentially the same: the modified VR is better than VR and introduction of a quark form factor greatly improves the results. In this case the VR approximation is poorer than the corresponding DNR analog. However, once a quark form factor is present, one obtains results of the same quality. Here again the dipole form does not affect the situation significantly.

Just to have a quantitative idea of the improvement, I give below in table 6 the chi square values calculated on all known decay widths (including the various leptonic channels) for the various studied formalisms.

\section{Conclusion}

In the framework of the NRQM concerning the meson wave function, and based on a field approach for the electromagnetic quark current operator, I study in this paper two different effects : the role of the kinematical relativistic corrections $\left(E_{p}\right.$ instead of $m$ ) and the influence of adding a quark form factor in the formalism. Two different phenomenological types for this form factor are tested : the monopole and the dipole form. Several observables are studied in the mesonic sector : the charge mean square radii, the form factors and the leptonic decays. Two different kinds of wave functions are considered : one resulting from a traditional Schrödinger equation, the other from a Salpeter equation.

The meson form factors depend only on the $F_{1}\left(q^{2}\right)$ quark form factor. The relativistic corrections improve a bit the theoretical results as compared to experimental data, but the main effect comes from the quark form factor. A monopole form is clearly favored. A nice agreement with data is obtained in that scheme without the need of a kinematical boost.

Concerning the leptonic decays, here too, the relativistic corrections improves the situation and a quark form factor gives a quite good agreement with data. The crucial parameter for explaining the decay is the quark anomalous magnetic moment. Monopole or dipole forms provide results of the same quality. These conclusions are independent of the internal dynamics of the quarks in- 
side the meson. The complete description of several observables in a unique consistent scheme seems to me a very important point.

The fact that, whatever the observable considered and whatever the wave function used, it is possible to find, for the electromagnetic quark form factor, a set of parameters that improves greatly the theoretical values convinces me that the existence of quasi particles inside meson is real and the physical description in terms of quark form factors makes sense and is very promising. However, a more fundamental explanation of this form factor, starting from basic QCD, is far from being obvious and needs certainly many other theoretical studies. It is probable that we can refine the model and tune the parameters by considering a larger bulk of physical observables.

\section{Appendix}

In this section, I want to show that the dynamical integrals that appear in the meson form factors can be calculated without too much difficulty. For that, I expand the $L=0$ meson radial wave function $\varphi(p)$ as a linear combination of gaussian functions. Thus I write the meson wave function in momentum representation :

$$
\Psi(\mathbf{p})=\frac{1}{\sqrt{4 \pi}} \sum_{i} C_{i} \exp \left(-\frac{A_{i}}{2} p^{2}\right)
$$

I have shown in several places [7]-[8] that an expansion up to 5 gaussians reproduces the exact wave function with a high degree of accuracy, so that using the right hand part of (41) instead of the true wave function does not make any difference on the results.

The NR bare square radius (21) is very easy to be obtained; it reads :

$$
\left\langle r_{N R}^{2}\right\rangle_{M}^{\text {bare }}=\frac{3 \sqrt{2 \pi}}{2\left(m_{\alpha}+m_{\beta}\right)^{2}}\left[e_{\alpha} m_{\beta}^{2}+e_{\beta} m_{\alpha}^{2}\right] \sum_{i, j} C_{i} C_{j} \frac{A_{i} A_{j}}{\left(A_{i}+A_{j}\right)^{5 / 2}}
$$

The $\mathrm{R}$ bare square radius (30) is more involved; it requires the evaluation of two elementary integrals

$$
I_{2}(A, m)=\int_{0}^{\infty} d p p^{2} \frac{\exp \left(-A p^{2}\right)}{\sqrt{p^{2}+m^{2}}} ; I_{4}(A, m)=\int_{0}^{\infty} d p p^{4} \frac{\exp \left(-A p^{2}\right)}{\sqrt{p^{2}+m^{2}}}
$$

Both are analytical and expressed in terms of modified Bessel functions; a very good numerical precision can be achieved. One has for the "relativized mass" 


$$
\frac{1}{\widetilde{m}_{\rho}}=\sum_{i, j} C_{i} C_{j} I_{2}\left(\left(A_{i}+A_{j}\right) / 2, m_{\rho}\right)
$$

and for the $K^{\rho}$ quantities appearing in (30):

$$
\begin{aligned}
\frac{K^{\rho}}{\widetilde{m}_{\rho}}= & \frac{3}{2} c_{\rho}^{2} \sum_{i, j} C_{i} C_{j} \\
& {\left[\frac{A_{i}+A_{j}}{2} I_{2}\left(\frac{A_{i}+A_{j}}{2}, m_{\rho}\right)-\frac{\left(A_{j}-A_{i}\right)^{2}}{6} I_{4}\left(\frac{A_{i}+A_{j}}{2}, m_{\rho}\right)\right] }
\end{aligned}
$$

The integral (18), which is the basic quantity of the NR meson form factor, is also very easy to be calculated. One finds explicitly :

$$
I^{\rho}\left(\mathbf{Q}^{2}\right)=\frac{\sqrt{\pi}}{4} \sum_{i, j} C_{i} C_{j}\left(\frac{2}{A_{i}+A_{j}}\right)^{3 / 2} \exp \left(-\frac{A_{i} A_{j}}{2\left(A_{i}+A_{j}\right)} c_{\rho}^{2} Q^{2}\right)
$$

The calculation of the integral (28) appearing in the relativistic expression of the meson form factor needs one more basic integral, namely

$$
I_{s}(A, B, m)=\int_{0}^{\infty} d p p \exp \left(-A p^{2}\right) \frac{\sinh (B p)}{B \sqrt{p^{2}+m^{2}}}
$$

As far as I know, it cannot be put under an analytical form, but a good precision for its numerical value can be achieved with classical algorithms. Let us note that it is an even function for the argument $B$. It can be shown easily that $\lim _{B \rightarrow 0} I_{s}(A, B, m)=I_{2}(A, m)$. Using this last property, the searched quantity is expressed as :

$$
\begin{aligned}
\frac{J^{\rho}\left(\mathbf{Q}^{2}\right)}{\widetilde{m}_{\rho}}=\sum_{i, j} C_{i} C_{j} I_{s}( & \left.\frac{A_{i}+A_{j}}{2}, \frac{c_{\rho}\left(A_{j}-A_{i}\right)}{2} Q, m_{\rho}\right) \\
& \times \exp \left(-\frac{A_{i}+A_{j}}{8} c_{\rho}^{2} Q^{2}\right)
\end{aligned}
$$

Thus, only using the convenient expression (41) for the wave function, I am able to calculate numerically, with a high accuracy, all the quantities needed for the evaluation of the meson form factor. 


\section{Acknowledgments}

I am indebted to F. Fernandez and A. Valcarce for useful discussions. I am particularly grateful to B. Desplanques for constant interest and for very fruitful discussions.

\section{References}

[1] D. Flamm, F. Schöberl, Introduction to the quark model of elementary particles, Gordon and Breach Science Publishers (1986)

[2] A. Le Yaounc, L. Oliver, O. Pène, J-C. Raynal, Hadron transitions in the quark model, Gordon and Breach Science Publishers (1988)

[3] L.P Fulcher, Phys. Rev. D50 (1994) 447

[4] C. Semay, D. Baye, M. Hesse, B. Silvestre-Brac, Phys. Rev. E64,(2001) 16703

[5] C. Semay, B. Silvestre-Brac, Nucl. Phys. A618 (1997) 455

[6] C. Semay, B. Silvestre-Brac, Nucl. Phys. A647 (1999) 72

[7] R. Bonnaz, B. Silvestre-Brac, Few. Body. Syst. 27 (1999) 163

[8] R. Bonnaz, B. Silvestre-Brac, C. Gignoux, Eur. Phys. J. A13 (2002) 363

[9] J.D. Bjorken, S.D. Drell, Relativistic quantum fiels, McGraw-Hill Book Company (1965)

[10] F.E. Close, An introduction to quarks and partons, Academic Press (1979)

[11] W. Greiner, J. Reinhardt, Quantum electrodynamics, Springer-Verlag (1992)

[12] B. Itzykson, J-B Zuber, Quantum field theory, McGraw-Hill, New York (1980)

[13] S. Weinberg, The quantum theory of fields, Cambridge University Press (1995)

[14] T. Ericson, W. Weise, Pions and nuclei, Oxford Science Publications (1988)

[15] B. Desplanques et al, Few. Body. Syst. 29 (2000) 169

[16] R. van Royen, V.F. Weisskopf, Nuovo. Cimento A50 (1967) 617

[17] L.A. Blanco et al, Nucl. Phys. A699 (2002) 690

[18] S.R. Amendolia et al, Nucl. Phys. B277 (1986) 168

[19] S.R. Amendolia et al, Phys. Lett. B178 (1986) 435

[20] G. Niculescu, Thesis, Hampton University (1998)

[21] Particle Data Group, Eur. Phys. J 15 (2000) 1 